\title{
A GAME THEORY CONTROL SCHEME IN MEDIUM ACCESS FOR WIRELESS BODY AREA NETWORK
}

\author{
Jing Zhou ${ }^{1,2}$, Aihuang Guo ${ }^{1}$, Juan $\mathrm{Xu}^{1}$, Hung Nguyen ${ }^{2}$, Steven Su ${ }^{2}$ \\ ${ }^{1}$ School of Electronics and Information Engineering, Tongji University, Shanghai, China \\ ${ }^{2}$ Faculty of Engineering and Information, University of Technology, Sydney, Australia \\ Jing.Zhou-2@student.uts.edu.au
}

Keywords: Wireless Body Area Network, Medium Access Control, Game Theory

\begin{abstract}
Wireless Body Area Network (WBAN) has been considered for applications in medical, healthcare and sports fields. Although there are several protocols for wireless personal area networks, specific features and reliability requirements in WBAN bring new challenges in protocol design. An appropriate control scheme in the MAC layer can make a significant improvement in network performance. Based on traffic priority and prior knowledge this paper proposes a game theoretical framework to smartly control access in contention period and contention free period as defined in IEEE 802.15.6 standard. The coordinator controls access probability of contention period based on users' priority in CSMA/CA and allocates suitable slots with strategies for best payoff based on link states in guaranteed time slots (GTS). The simulation results show the improved performance especially in heavily loaded channel condition when the optimal control mode is applied.
\end{abstract}

\section{Introduction}

Wireless Body Area Network (WBAN) has raised interest in many areas, such as: medical scenarios, healthcare centres, sports fields and so on. Although there are several standards for wireless personal area networks, they are not adequate to satisfy WBANs' high reliability, energy efficiency and other specific features. IEEE 802.15 Task Group 6 designs a short range and low power wireless communication standard on, in or around body area [1] The performance of this protocol is evaluated by many researchers. Yufei Wang evaluated the proposal under WiFi/Bluetooth interference in medical multi-parameter monitoring [2]. Ullah, S analyzed the throughput and delay performance of the IEEE 802.15.6 [3]. Actually the standard just outlines the basic elements to ensure interoperability and leaves open questions for appropriate control at the MAC layer [4]. Successful systems are highly dependent on the characteristics of applications and need to be adaptive with a dynamical control and configuration. So it is still necessary and essential to develop an optimal method for better performance.

In IEEE 802.15.6 protocol contention period and contention free period are defined in Medium Access Control
(MAC) layer. Contention access applies CSMA/CA for narrowband while contention free access uses scheduled access with guaranteed time slots. Collisions are associated with the channel access probability, which is decided by the back off algorithm on contention window in CSMA/CA. Avoiding collisions is a prior issue in designing MAC protocol because it is critical to improve system performance. There is a wealth of research to concern this issue. Shih, Kuei-Ping proposes an adaptive range-based power control MAC protocol to prevent collision [5] Fang designs two collision-free access schemes with optimal long-run throughput [6]. The contention free access uses guaranteed slots to obtain scheduled uplink, downlink, and bi-link allocations. But it still leaves questions on efficient allocation and there is limited research on this issue. Beside CSMA/CA, for scheduled access scheme (GTS) traffic priority and prior knowledge of the network status can be used for optimization. So an appropriate control scheme in the MAC layer can make a significant progress in performance.

In this paper we propose a novel game theory framework for collision avoidance and slots allocation in WBANs. Game theory is a mathematic method to study the interactions between several decision-makers who have conflicts or common interest. A game model comprises a set of players, which choose their actions in each period of the game to maximize that period's expected payoff. Payoff usually represents profit, quantity, "utility," or other continuous measures or simply illustrates the desirable outcomes. Game theory has been used in communication protocol for better payoff, for example: throughput, latency, energy and so on. R. Machado surveys the use of game theory to solve the problems of energy efficiency, security and pursuit-evasion games in sensor networks [7].

The rest of this paper is organized as following: Section 2 briefly introduces the MAC protocol and user priority of IEEE 802.15.6. Section 3 describes the game model of CSMA/CA with the definition of utility function, strategies and Nash Equilibrium for better payoff. Section 4 illustrates the game model of scheduled allocation. Section 5 presents the simulation and explains results. Section 6 makes a conclusion.

\section{Overview of MAC Layer in IEEE 802.15.6}

\subsection{Access Mechanisms}

In 802.15.6 the access mechanisms are divided into three categories: 1) Random access mechanism, which uses 
either CSMA/CA or a slotted Aloha procedure for resource allocation; 2) Improvised and unscheduled access, which uses unscheduled polling/posting for resource allocation; 3) Scheduled access and variants (connectionoriented contention-free access), which schedules the allocation of slots in one or multiple upcoming superframes, also called 1-periodic or m-periodic allocations.

CSMA/CA and scheduled allocation of guaranteed time slots are widely accepted in applications. But the default configuration does not capture the real situation and behavior for high quality of service. Based on the perceived network cognitive knowledge and traffic priority, we apply game theory models to dynamically control these two mechanisms for high reliability and better performance.

\subsection{User Priority}

In 802.15.6 user priority (UP) values, which can be referenced in prioritizing medium access of data and management type frames, will be determined based on the designation of traffic according to Table 1. So there are eight priority values for different traffic types.

\section{Game Theory Model in CSMA/CA}

\subsection{CSMA/CA Scheme}

We design our access scheme based on the CSMA/CA standard, which applies the Random back-off time algorithm to reduce the probability of collisions. Each node in the network can sense the channel before transmitting the data packet. Sensing the channel means to monitor the status of channel whether it is clear or busy. If the channel is clear, it means no other node is transmitting at this time. If the channel is clear, then the expected packet is sent. If the channel is busy, the node waits for a randomly period of time, and then checks again to see if the channel is clear. This waiting period is called back--off time and counted down by a back-off counter. A node sets its back-off counter to a random integer number uniformly distributed over the interval $[1, C W]$ where $C W$ is called contention window and $C W \in\left[C W_{\min }, C W_{\max }\right]$. The value of $C W$ is

Table 1. User priority mapping.

\begin{tabular}{ccc}
\hline User Priority & Traffic designation & Frame type \\
\hline 0 & Background & Data \\
1 & Best effort & Data \\
2 & Excellent effort & Data \\
3 & Video & Data \\
4 & Voice & Data \\
5 & Controlled load & Data/management \\
6 & Media data/network control & Data/management \\
7 & Emergency/medical event report & Data \\
\hline
\end{tabular}

doubled for an even number of failures (when the node fails to receive an acknowledgement or group acknowledgement) until it reaches $C W_{\max }$. The back-off algorithm in CSMA/CA just tries to avoid collisions, but could not remove it all. The UPs in IEEE 802.15.6 indicate the priorities for access to the medium but this protocol only simply set the contention window. It is necessary to adjust back-off time dynamically by a constrained optimization method.

\subsection{Random Access Game Model}

A game model is proposed in random access by controlling the back off time. We consider a body area network with $\mathrm{n}$ wireless nodes, which always have a frame to transmit and are synchronized to support the contentionbased mechanism in IEEE 802.15.6. A coordinator or any nodes may obtain contended allocations in EAP (Exclusive Access Phase) 1 and EAP2 if it requires the transmission of data frames. A non-cooperative game model is used for access control. The non-cooperation game reflects a competitive situation where every player needs to take its decision independently of other players, giving the possible choices of other players and their effect on the players' utilities.

Definition 1: A general random access game $\mathrm{G}$ is defined as a triple $G=\left\{N,\left(S_{i}\right)_{i \in N},\left(U_{i}\right)_{i \in N}\right\}$, where $N=\{1, \ldots, n\}$ is the finite set of players, $S_{i}$ is the available strategies and $U_{i}$ is the utility function to evaluate the payoff. In our analysis $\mathrm{N}$ represents wireless nodes in the body area network. Choosing the contention window is the strategy that every node uses. The payoff is the throughput, which is an important measurement to evaluate the performance. It is denoted as the average fraction of time that the radio channel takes to successfully carry packets.

Since we assume that a player's object is to maximize his throughput (and we assume he always has a packet to send), he will tend to use the full channel capacity. We make use of the same model as Bianchi to estimate the throughput in a two-dimensional Markov chain of $m$ backoff stages [8]. A transition takes place upon collision to a higher stage or successful transmission to the first stage respectively.

Assumption: Nodes (sensors) are players in this game and all nodes make their decision simultaneously. The utility function (throughput) of node $\mathrm{i}$ is defined as:

$$
U_{i}=P_{s i} \times L \times A
$$

where $\mathrm{A}$ is a normalization constant to convert to bps, $\mathrm{L}$ is the payload, $P_{s i}$ is the probability that any node successfully transmit in contention period. The strategy can be choosing contention window which is related to the access probability. The successful access probability of node $i$ is defined in as:

$$
P_{s i}=\tau_{i}\left(1-\tau_{i}\right)^{n-1},
$$

where $\tau_{i}$ is the access probability of node $i$. It depends on the contention window $\mathrm{CW}$ as in (3): 


$$
\tau_{i}=2 /\left(C W_{i}+1\right) .
$$

Considering users' priority the contention window is multiplied by the priority factor $\eta_{i}$, we use normalized values with respect to each sensor's specific constraints and get:

$$
\tau_{i}=2 /\left(\eta_{i} C W_{i}+1\right) .
$$

In a complete access game one of the most important problems is the solution of Nash Equilibrium, which is a state of non-cooperative game where no player can improve its utility by changing its strategy, if other players maintain their current strategies.

Definition 2: The vector $s^{*}$ is a Nash Equilibrium if $\forall i \in K \quad \forall s_{i} \in S_{i}, u_{i}\left(s_{i}^{*}, \underline{s}_{-i}^{*}\right) \geq u_{i}\left(s_{i}, \underline{s}_{-i}^{*}\right)$. A Nash equilibrium $s_{i}^{*}$ is a nontrivial equilibrium if $s_{i}^{*}$ meets $\frac{\partial}{\partial p_{i}} u_{i}\left(s_{i}^{*}, s_{-i}^{*}\right)=0, \forall i \in K$ So a necessary condition for a local maximum existing is:

$$
\frac{\partial}{\partial C W_{i}} u_{i}\left(s_{i}^{*}, s_{-i}^{*}\right)=\frac{\partial}{\partial C W_{i}}\left[\frac{2}{\eta_{i} C W_{i}+1}\left(\frac{\eta_{i} C W_{i}-1}{\eta_{i} C W_{i}+1}\right)^{n-1}\right]=0 .
$$

Now there are two solutions: $C W_{i}=\left\lfloor 1 / \eta_{i}\right\rfloor$ and $C W_{i}=\left\lfloor(2 n-1) / \eta_{i}\right\rfloor,\lfloor\rfloor$ means the floor integer value because the contention window should not be decimal. The individual utility of each sensor depends on the strategies of selecting a set of windows to maximize the utility of a system.

1) For first solution when it is emergency situation we set $\eta_{i}=1$ and the $C W_{i}=1$. It means the access probability is $\tau_{i}=1$ and this node capture the whole channel. At that time this node reaches the maximum throughput and others nodes' throughputs are zero. This strategy can be used for special situation because only one node gets positive payoff and other nodes sacrifice their payoff. But it is unfair for all nodes in the whole network at normal situation.

2) When we choose $C W_{i}=\left\lfloor(2 n-1) / \eta_{i}\right\rfloor$, the solution can bring a fair distribution and the system can acquire optimal configuration. In association with the amount of nodes in a network every node gets the maximum available access probability with orderly back-off time and avoids collisions. This solution is more appropriate in normal operation.

\section{Game Theory Model in Scheduled Access}

\subsection{Scheduled Allocation Mechanism}

IEEE 802.15.6 defines the scheduled allocation access (Contention free) with two modes: 1-periodic where devices exchange frames with the coordinator in every superframe or m-periodic where devices and the coordinator exchange frames every $\mathrm{m}$ superframes allowing the device to sleep between transfers. In scheduled access mechanism devices can start their transfer when the reserved allo- cation slot time has commenced.

Using scheduled access can avoid collisions but it is not flexible so the optimal scheduling techniques are stillnecessary. Unsuitable allocation can cause the loss of balance. At certain situation some users' resources may be redundant for their QoS while other users' requirements may not be guaranteed. In worst case emergency data have to wait in a long queue and eventually fail to transmit. It is a challenge to choose an adaptive slot allocation scheme to increase the reliability and efficiency. Usually users want to obtain sufficient slots for a large amount of data but a divisible resource is shared by all nodes. If the allocated slots cannot meet QoS the coordinator should adjust slots in an optimal method.

Prior knowledge or cognitive knowledge is the status of every member in network from hardware level to protocol stack and can provide the information of QoS. Combined with game-theoretic mechanisms cognitive knowledge can be useful to optimize the multi-users slots allocation problem. With the observed link state, a dynamic slots allocation game scheme is designed in our research to model users' competition and be services-dependant. In the scheme, the coordinator calculates a solution for an optimal policy, which is the action of maximum profit.

Here the profit is the system throughput $S(t)$ of the superframe $t$ is given by the ratio of the average length of successfully allocated payload in GTS slots to the average length of time in Equation (6):

$$
S(t)=\frac{P_{s}(t) \times L_{p l} \times \tau_{n} \times N_{t s}}{\text { QLatency }+T_{s}}
$$

where $P s(t)=1-\operatorname{Pbreak}(t), \operatorname{Pbreak}(t)$ is the ratio of breakdown packets, $\mathrm{Lpl}$ is the length of payload of each data packet, $\tau_{\mathrm{n}}$ is the number of data packets in one slot, $N_{t s}$ is the number of superframe slots. QLatency is the queuing latency, which is related to the allocated slots for each node, and Ts is the transmission time in slots.

\subsection{Scheduled Access Game Model}

As discussed in section 3, a scheduled access game $\mathrm{G}$ is defined as a triple set $G=\left\{N,\left(S_{i}\right)_{i \in N},\left(u_{i}\right)_{i \in N}\right\}$. Total amount of available slots shared among $\mathrm{N}$ players is denoted as B, the coordinator determines slots as strategies to acquire maximum payoff of throughput. User i's utility function of payoff is defined as:

$$
u_{i}=f\left(s_{i}\right)+g_{c}\left(s_{i}\right),
$$

$f\left(s_{i}\right)$ represents the basic throughput, which is a increasing, nonnegative function of node's slots. $g_{c}$ is the compensation function associated with the services satisfaction in (8) when the total slots is lower than the maximum available slots.

$$
g_{c}=p\left(s_{i}\right) \times \max \left\{0, P B_{i}(t)-E_{i}\right\} .
$$

$p\left(s_{i}\right)$ is a parameter to determine slots, $\mathrm{PB}$ is the actual packets breakdown of node i, $E_{i}$ is the maximum ac- 
cepted packets breakdown in required QoS. If $P B_{i} \leq E_{i}$, it means the allocated slots meet the QoS and the compensation function doesn't work with

$p\left(s_{i}\right) \max \left\{0, P B_{i}(t)-E_{i}\right\}=0$. Otherwise the coordinate will trigger the compensation function as

$p\left(s_{i}\right) \max \left\{0, P B_{i}(t)-E_{i}\right\}=p\left(s_{i}\right)\left[P B_{i}(t)-E_{i}\right]$ and allocate more slots to obtain best payoff. User i's optimization problem in the game would be: $\max \left[f\left(s_{i}\right)+g_{c}\left(s_{i}\right)\right]$. The optimal slots vector is written as $s^{*}$, then the solution is $U_{i}^{*}=\arg \max \left[f\left(s_{i}^{*}\right)+g_{c}\left(s_{i}^{*}\right)\right]$ to one player. For the whole network $U^{*}=\arg \max \sum\left[f\left(s_{i}\right)+g_{c}\left(s_{i}\right)\right], U^{*}$ is the Nash equilibrium when players obtain the best strategies with given slots. The constraint is the total slots are less than B.

\subsection{Procedure of the Game}

In a star network, all $\mathrm{N}$ nodes send data to the coordinator, which is the data sink. Firstly, the coordinator allocates slots according to basic requirements. Then it observes network status and acquires information. If the QoS is not good, such as the amount of breakdown packets is higher than a threshold the coordinator implements the game to change slots allocation. The game process continues until the system reaches the equilibrium point of the best payoff or the maximum amount of slots. A dynamic and adaptive scheduling technique can increase the throughput by reducing packets failure and delay.

\section{Simulation and Analysis}

The optimal access control models are verified by simulations with the open source simulator Castalia 3.2. The network topology is assumed as a star structure because it is common in WBAN. All nodes send data packets (128 bytes/ packet) to one coordinator. The wireless transceivers operate in $2.4 \mathrm{GHz}$ band with data rate of $1024 \mathrm{kbps}$. Receiver sensitivity is $-87 \mathrm{dBm}$ and the transmit power is $-10 \mathrm{~dB}$. Payoff is defined as throughput in the game theory scheme.

Firstly we compare the payoff between the proposed optimal model and a random mechanism of CSMA/CA. In this simulation, five sensor nodes send data to one coordinator at different data rate (25 packets, 50 packets and 100 packets per second). As shown in Figure 1 nodes achieve more throughputs by using the new mechanism with game theory (shown as optimal) than the random mechanism (shown as standard).

Figure 2 shows that with the smallest contention window the payoff of Node 5 increases substantially because of its highest priority at the sacrifice of other nodes. Furthermore, the throughput obtained by each node in optimal model increases monotonically when the traffic load is high while throughputs of some nodes decrease due to collisions in standard model.

Then we apply the game algorithm to one coordinator and fourteen nodes in a star network. As shown in Figure 3

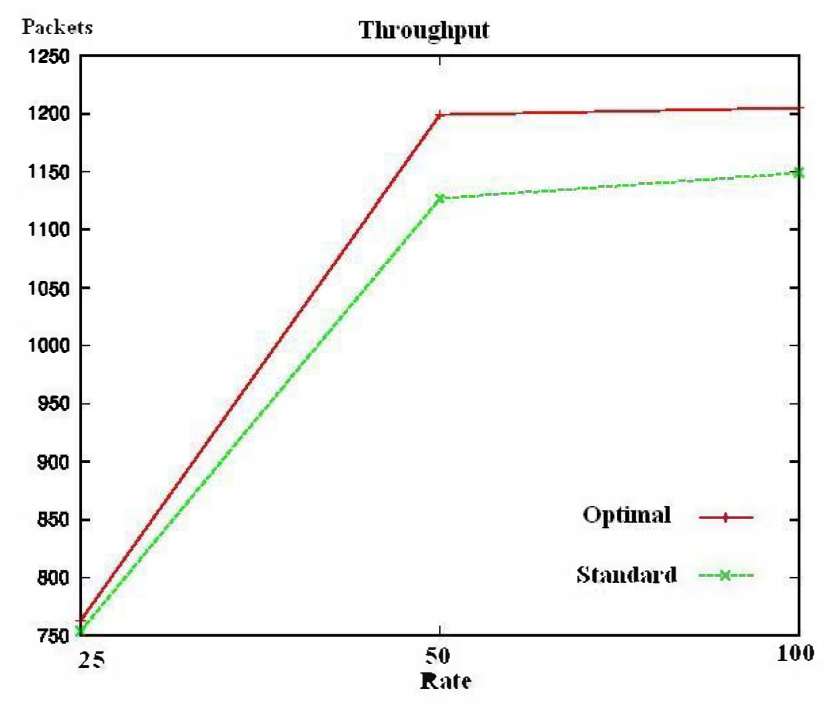

Figure 1. Throughput of nodes in contention access.

$$
\text { Packets Received Per Node }
$$

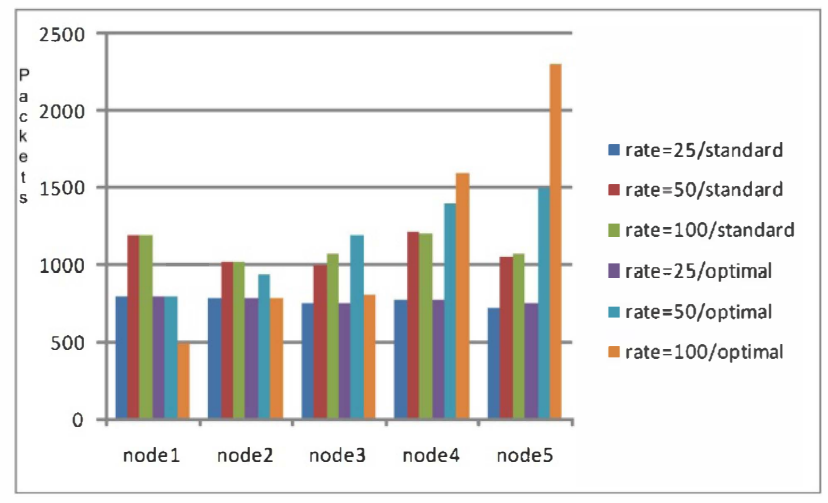

Figure 2. Packets of 5 nodes in contention access.

players (such as node 12, 14 and etc.) with high priority and small contention window receive better payoff when other nodes use large contention windows.

The observation can be explained as following: when the system works at high data rate or many nodes, the amount of nodes competing for access at a certain time may be large and new nodes continuously enter the set of competing nodes. Therefore heavily traffic load causes more collisions. The optimal model can adjust the contention window to reduce the collision and improve the whole system throughput.

Then we explore the performance of scheduled access game in a network of one coordinator and ten nodes at different data rate (20 packets, 50 packets and 100 packets per second) when the standard allocation can't meet the QoS. Here the packets breakdown is observed at the MAC layer as criteria to trigger the game theory scheme. When the amount of dropped packets is higher than the maximum accepted number, the coordinator schedules the slots until it reaches the Nash equilibrium with best strategies, which means more slots are allocated for high throughput.

Figure 4 shows the total throughput of scheduled access with optimal control and standard control. When allocated slots are not enough at high speed the throughput 


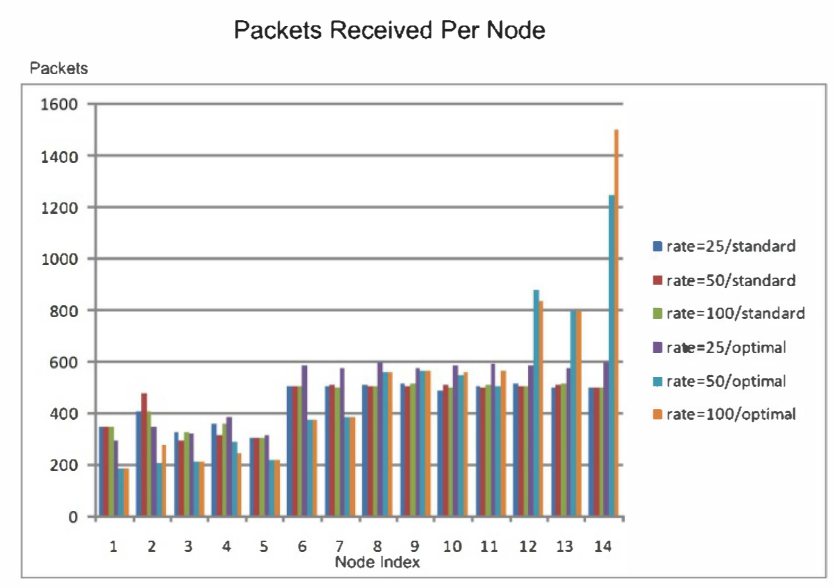

Figure 3. Packets of 14 nodes in contention access.

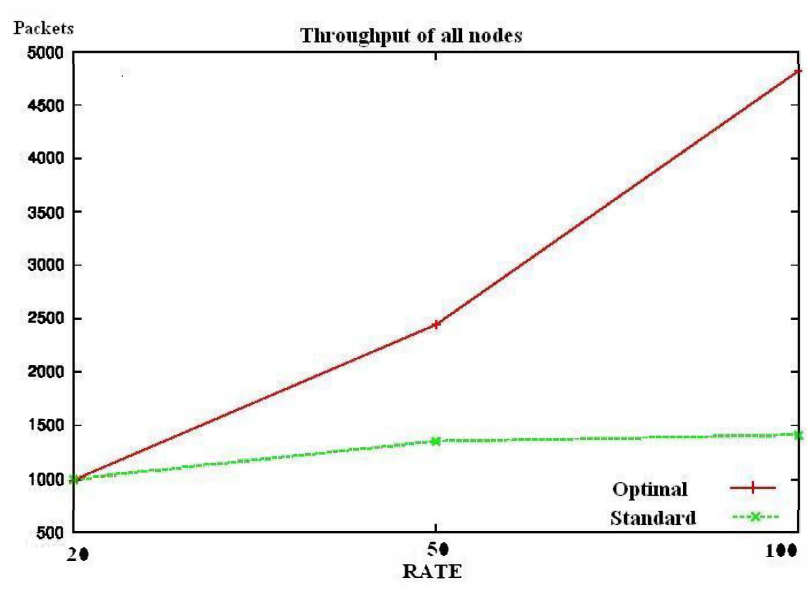

Figure 4. Throughput of 10 nodes in Scheduled access.

does not increase. By detecting breakdown packets the best strategy is calculated for an optimal slot allocation. It is obvious to see the optimal model increases the payoff dramatically especially at high data rate due to the smart game scheme. At that time each node gets enough slots to meet their QoS requirement.

The amount of breakdown packets reduces as shown in Figure 5. When data rate is low the basic allocated slots can meet the QoS. While data rate is high the breakdown packets increase and the coordinator allocate more slots to nodes. Then the amount of dropping packets reduces and the system gets best throughput.

\section{Conclusion}

This paper designs a game theoretical framework to automatically configure parameters for better throughput in contention access based on priority and contention free access based on cognitive information. In contention access period the game theory algorithm increases the access probability by controlling of contention window and in contention free period nodes are allocated maximum available slots to meet the QoS. The simulation results show the performance improves in optimal model especially at heavily loaded channel condition. There are still a lot of work to do for high reliability and good performance

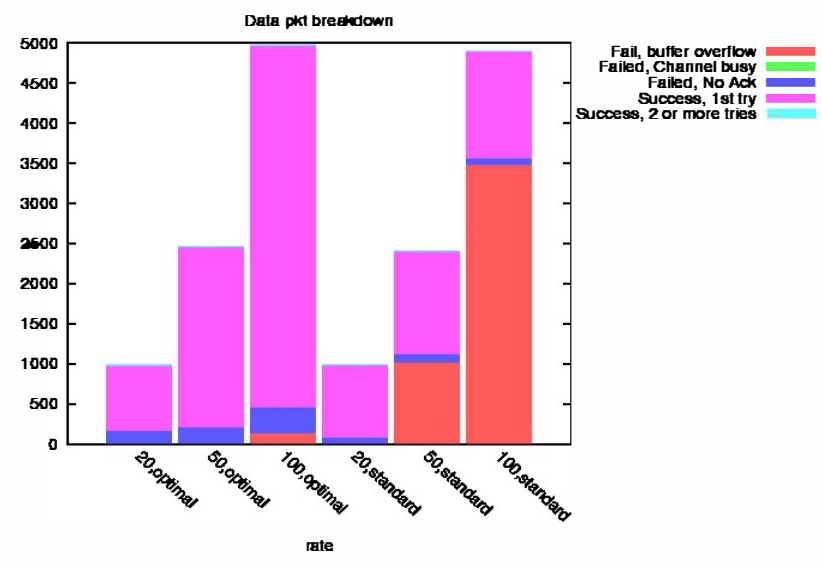

Figure 5. Breakdown packets in two schemes at 3 rates.

of WBAN in applications. For example: cooperation of nodes in the network, a dynamic model with considering more cognitive knowledge, intelligent control of scheduled and improvised access for high efficiency services and better resource utilization. These issues will be considered in the future research.

\section{Acknowledgements}

Research partly supported by National Natural Science Foundation of China under Grant No. 61202384.

\section{References}

[1] Association, I.S. (2012) IEEE Standard for Local and Metropolitan Area Networks, in Part 15.6: Wireless Body Area Networks. IEEE Computer Society, New York.

[2] Wang, Y. and Wang, Q. (2011) Evaluating the IEEE 802.15.6 2.4GHz WBAN Proposal on Medical MultiParameter Monitoring under WiFi/Bluetooth Interference. Journal of E-Health and Medical Communications, 2, 48-62.

http://dx.doi.org/10.4018/jehmc.2011070103

[3] Ullah, S., Chen, M. and Kwak, K.S. (2012) Throughput and Delay Analysis of IEEE 802.15.6-Based CSMA/CA Protocol. J Med Syst, 36, 3875-3891.

http://dx.doi.org/10.1007/s10916-012-9860-0

[4] Boulis, A., et al. (2012) Challenges in Body Area Networks for Healthcare. IEEE Communication Magazine, 50, 100-106.

http://dx.doi.org/10.1109/MCOM.2012.6194389

[5] Shih, K.-P., Chen, Y.-D. and Chang, C.-C. (2011) A Physical/Virtual Carrier-Sense-Based Power Control MAC Protocol for Collision Avoidance in Wireless Ad Hoc Networks. IEEE Transactions on Parallel \& Distributed Systems, 22, 193-207. http://dx.doi.org/10.1109/TPDS.2010.75

[6] Fang, M., et al.(2013) Decentralised Learning MACs for Collision-Free Access in WLANs. Wireless Networks, 19, 83-98.

[7] Machado, R. and Tekinay, S. (2008) A Survey of Game-Theoretic Approaches in Wireless Sensor Net- 
works. Computer Networks, 52, 3047-3061.

http://dx.doi.org/10.1016/j.gaceta.2008.07.003

[8] Bianchi, G. (2000) Performance Analysis of the
IEEE 802.11 Distributed Coordination Function. Selected Areas in Communications, 18, 535-547.

http://dx.doi.org/10.1109/49.840210 